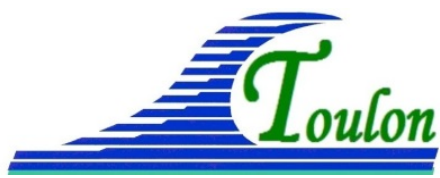

XIV èmes Journées Nationales Génie Côtier - Génie Civil

Toulon, 29 juin au $1^{\text {er }}$ juillet 2016

DOI:10.5150/jngcgc.2016.084 (C) Editions Paralia CFL

disponible en ligne - http://www.paralia.fr - available online

\title{
Suivi des activités d'extraction du sable marin au Maroc
}

\author{
Mounir HAKKOU ${ }^{1}$, Aïcha BENMOHAMMADI ${ }^{2}$, \\ Bruno CASTELLE ${ }^{3}$, Hind AZIDANE ${ }^{4}$
}

1. University Mohammed V in Rabat, Institut Scientifique, Département des sciences de la terre, Laboratoire GEOTEL, BP 703, Rabat-Agdal, Maroc.

m.hakkou@gmail.com

2. Université Ibn Tofeil, Faculté des sciences, Département des sciences de la terre, B.P. 133, Kénitra, Maroc.

3. Université de Bordeaux, CNRS, UMR EPOC 5805, Talence, F-33405, France.

4. Université Ibn Tofeil, Faculté des sciences, Département des sciences de la terre, B.P. 133, Kénitra, Maroc.

\section{Résumé :}

Le dragage des matériaux marins au niveau des estuaires et en offshore au Maroc pour les besoins d'entretien et/ou de construction soulèvent des questions relatives à la gestion de leurs impacts sur l'environnement ainsi que la stabilité du littoral et des ouvrages qu'il héberge. Ce travail donne un aperçu sur les impacts physiques et écologiques des projets de dragage du sable marin et apporte quelques mesures techniques pouvant être prises en considération lors de l'évaluation de leurs études environnementales.

Mots-clés : Dragage des matériaux marins, Impacts physique et écologique, Evaluation environnementale.

\section{Introduction}

Les activités de dragage d'exploitation du sable marin sont aujourd'hui en pleine évolution au Maroc. Elles attirent de plus en plus l'attention des opérateurs de dragage, notamment dans les milieux estuariens et au large. Les impacts de ces activités sur le milieu naturel sont multiples, et peuvent être physiques, chimiques et biologiques. L’importance et l'intensité de ces impacts dépend des composantes et objectifs du projet de dragage (entretien, exploitation, ...) et de la typologie et la nature des milieux sédimentaires visés par ces activités (estuaire, lagune, mer, ...) ainsi que les facteurs de forçages (courants, vagues, bathymétrie, ...). L’absence au Maroc d'un cadre juridique réglementant ces activités a motivé la réflexion sur les mesures techniques principales à considérer dans leur évaluation environnementale. Le présent article s’inscrit dans cette perspective. Il apporte dans un premier temps un aperçu sommaire sur les principaux impacts pouvant être observés lors du dragage d'exploitation et/ou d'entretien dans les milieux estuariens et au large, et discute dans un second temps les éléments principaux à considérer lors de l'évaluation environnementale de ces projets. 


\section{Principaux impacts du dragage du sable marin}

\subsection{Impacts physiques}

L'impact généré dépend de nombreux facteurs, notamment la méthode et l'intensité de l'extraction, le type et la mobilité des sédiments, la topographie du fond, et l'intensité du courant (NEWELL et al., 1998). La modification de la bathymétrie induit des changements de l'hydrodynamique des vagues et des courants qui agissent à leur tour sur le régime sédimentaire au droit de la zone d'extraction et au-delà de celle-ci, notamment sur les conditions d'érosion et de sédimentation des côtes sableuses lorsque le dragage s'effectue dans de faibles profondeurs (TILLIN et al., 2011). Ces impacts agissent donc non seulement sur l'évolution et la restauration de la zone d'extraction, mais aussi sur le recul du trait de côte car les sables apportés par la houle et les courants, qui contribuent à l'équilibre sédimentaire des côtes, sont en partie piégés dans les excavations laissées par les dragues. C'est pour cela qu'il est nécessaire de définir une profondeur limite d'extraction suffisamment loin de la côte pour que les sédiments des plages mobilisés par les courants et par la houle ne viennent pas se piéger dans les excavations de dragage, et qu'ils puissent revenir à la plage pour alimenter son équilibre sédimentaire. Un exemple classique est celui de la destruction du village de Hallsands, dans le Devon, au cours du $20^{\text {ème }}$ siècle où le dragage a été effectué à proximité de la plage, ce qui a entraîné par la suite sa forte érosion (CIRIA, 1998). Au Maroc, le dragage intensif de l'embouchure de l'oued Sebou pour les besoins d'entretien des chenaux de navigation mais aussi pour l'exploitation du granula, est suspecté d'être à l’origine de l'érosion du littoral de Mehdia (DPDPM, 2011).

\subsection{Impacts écologiques}

Le dragage des estuaires ne se fait pas uniquement dans le seul objectif du maintien du tirant d'eau nécessaire pour faciliter la navigation. Il peut aussi faire l'objet de production du granula. Les matériaux de ces milieux sont plus ou moins chargés en contaminants (produits organiques, métaux) provenant des activités urbaines, industrielles et portuaires avoisinantes. La turbulence générée par l'extraction sur le fond remobilise les sédiments fins de la couverture sédimentaire et par conséquent affecte les conditions physico-chimiques de l'eau et des habitats. Les travaux de dragage peuvent entrainer une réduction de 30 à $70 \%$ de la diversité des espèces (species diversity), 40 à 95\% le nombre d'individus (number of individuals) et autant pour la biomasse des communautés benthiques (NEWELL et al., 1998).

Les extractions remuent les polluants déposés sur plusieurs années sur les fonds et les rediffusent dans la chaine alimentaire. La turbidité générée asphyxie les milieux et porte atteinte aux espèces benthiques et pélagiques, notamment celles caractérisées par leur faible mobilité (GEODE, 2012). Un étalement sur le fond d'une partie des suspensions 


\section{XIVìmes Journées Nationales Génie Côtier - Génie Civil \\ Toulon, 29 juin au $1^{\text {er }}$ juillet 2016}

sous la forme d'un courant de densité s'étend jusqu'à $800 \mathrm{~m}$ dans le sens des courants et sur 3 à 4 m d'épaisseur pour une surverse par puits (HITCHCOCK\& BELL, 2004), et peut s'étendre sur plusieurs $\mathrm{km}$ de long, plusieurs centaines de mètres de large (DICKSON\& REES, 1998 ; HITCHCOCK et al., 1999 ; NEWELL et al., 1999). Cela dépend de la vitesse du courant, du débit rejeté, de la nature du sédiment, etc. Certains auteurs montrent que des concentrations anormales persisteront plusieurs heures après le rejet (WILLOUGHBY\& CRABB, 1983). Malgré des concentrations tendant rapidement vers celles du milieu naturel, ce panache peut être encore visible 1h30mn après le rejet (BOUTIMIN, 1986).

L’importance de ces impacts et la possibilité de revenir à un état proche de l'état initial après les extractions sont surtout fonction de l'intensité de l'extraction et de la résilience écologique du site. Ils dépendent également des objectifs de travaux, de la stratégie et des procédés d'exploitation proposés, des conditions morpho-bathymétriques et sédimentaires du site ainsi que de l’hydrodynamisme local.

\section{Les mesures techniques à considérer}

Pour veiller sur la stabilité physique du littoral et préserver les écosystèmes marins de la dangerosité de la rediffusion des polluants générés par les panaches turbides lors des travaux de dragage, plusieurs précautions sont à considérer. Celles qu'on présentera ciaprès ne constituent qu'une première ébauche pouvant être développée selon les spécificités des zones d'extraction. Ces précautions peuvent constituer un fondement technique non exhaustif pour la conception d'une directive traitant l'évaluation de l'aspect environnemental de cette activité au Maroc. Au moins cinq mesures nous semblent indispensables à aborder (HAKKOU et al., 2015).

\subsection{L'étude d'impact environnemental}

Compte tenu des spécificités physiques et écologiques des zones d'extraction et des composantes des projets de dragage, une étude d'impact environnemental préalable s'impose dans l'objectif d'atténuer les différents types d'impacts, de les compenser ou de proposer d'autres alternatives moins dégradantes. Cette étude est tenue de : (i) définir les caractéristiques physiques et biologiques du milieu naturel et leurs variabilités spatio-temporelles (bathymétrie, sédimentologie, courants, biocénose...), (ii) étudier les impacts directs et indirects engendrés par l'excavation générée par le passage de l'élinde, le panache et le dépôt de ses particules, et le criblage, (iii) étudier l'impact physique au-delà de la zone d'extraction, notamment les conditions de propagation des vagues, changement des courants et modifications morphologiques des côtes, et (iv) analyser la restauration du milieu suite à ces impacts. Le point (iii) est abordé dans certains pays comme l'Angleterre par des simulations des conditions maximales de l'agitation de la mer (TILLIN et al., 2011) : lorsque la modélisation des extractions prédit des changements de plus de $2 \%$ dans les conditions de propagation de la houle à 


\section{Thème 6 - Gestion durable des zones littorales et estuariennes}

proximité immédiate de la zone d'extraction, le projet d'extraction n'est pas autorisé et l’opérateur est invité à réviser soit les composantes et le plans de son projet, soit la zone d'extraction.

L'étude d'impact environnemental constitue aussi un outil technique adapté pour la proposition de plans de suivi et de surveillance pour toutes les filières de cette activité. Pour cela, une cartographie des gisements du granulat, des zones conchylicoles doit être établie avant le démarrage de l'étude d’impact environnemental.

\subsection{La profondeur limite d'extraction du sable marin}

Les changements opérés sur la morphologie des fonds influencent généralement la propagation des vagues et par conséquent le transport sédimentaire qui conditionne l'évolution morphologique de la côte. Lorsque l'extraction du sable est effectuée au large, la fosse résultant de l'extraction du sable marin peut migrer vers le rivage et agir comme un puits pour les sédiments du système littoral (VAN RIJN \& WALSTRA, 2002). La gravité et la persistance de ces effets dépendent, entre autres facteurs, des conditions hydrodynamiques, de la nature du substrat, de la géométrie de la zone de dragage et de sa distance à la côte, et de l'échelle de temps de la perturbation des fonds marins (COOPER et al., 2007 ; ROOS et al., 2008). Si l'extraction est opérée dans la zone de déferlement, l'impact est massif et immédiat. En dehors de cette zone, il s'atténue rapidement quand la souille s'éloigne du rivage. Afin d'atténuer l'impact de l'érosion de la côte, une des démarches adoptée est de situer la zones de dragage au-delà d'une profondeur dite de fermeture qu'on peut déterminer par diverses équations. Le modèle empirique de HALLEMEIER (1981) par exemple définit cette profondeur $\left(\mathrm{h}_{\mathrm{l}}\right)$ par l'équation:

$$
h_{1}=2,28 H_{s e}{ }^{2}-68,5\left(\frac{H_{s e}{ }^{2}}{g T_{e}{ }^{2}}\right)
$$

Où $H_{s e}$ et $T_{e}$ sont respectivement la hauteur significative des vagues de tempête qui n'est pas dépassée plus de $12 \mathrm{~h}$ par an (occurrence de 0,137 \%) et la période des vagues associée, et g l'accélération de la gravité. Cette profondeur est mobile à l'échelle annuelle et interannuelle, sous l'action des houles de tempêtes (DESMAZES et al., 2002). A Kénitra (Maroc), pour une hauteur significative de $7,76 \mathrm{~m}$ et une période de pic de 16,43 s, dépassée en 12 heures en 30 janvier $1998\left(H_{S m a x}=8,25 \mathrm{~m} ; T=16,53 \mathrm{~s}\right.$; données de WW3), on estime cette profondeur à 16,05 m. Par ailleurs, la forte agitation qui a affecté les côtes marocaines en date du 6 et 7 janvier 2014, dont la houle avait dépassée à plusieurs reprises le pic de $13 \mathrm{~m}\left(H_{\max }=13,62 \mathrm{~m}\right.$; T=21,6 s) au large de la ville de Mohammedia (METL 2014), incite à revoir à la hausse cette estimation. Une autre approche de détermination de cette profondeur de fermeture est fondée sur le suivi de haute résolution des profils bathymétriques sur plusieurs années (DESMAZES et al., 2002 ; JAUD, 2011). 


\section{XIV èmes Journées Nationales Génie Côtier - Génie Civil \\ Toulon, 29 juin au $1^{\text {er }}$ juillet 2016}

\subsection{L'éloignement des zones conchylicoles}

Les activités de dragage doivent être suffisamment éloignées des sites de valeur conchylicole importante pour éviter leur asphyxie et contamination. La distance minimale séparant le site d'extraction du sable de ces milieux, doit prendre en considération l'aire de l'extension et de dépôt du panaché turbide. Celui-ci, comme a été indiqué en haut, dépend des techniques et procédés d'extraction, de la nature du sédiment (sable grossier, moyen ou fin; teneur du silt,...), des conditions hydrodynamiques (courants), de l'intensité du dragage, ... Des études de simulation de la turbidité peuvent aider à la détermination de cette distance suivant les spécificités de chaque projet de dragage.

\subsection{Le suivi et l'audit des déclarations}

Le suivi pendant la phase d'exploitation qui peut se faire à distance et en temps réel favorisé par les nouvelles technologies GPS, permet de s'assurer du respect des limites géographiques et volumétriques autorisées pour l'exploitation (tracé des zones de dragage, profondeur, tonnage, ...). L'établissement de levés bathymétriques des zones de dragage, avant et après l'exploitation, servent de référence pour le suivi et le contrôle des opérations d'extraction du sable marin. Les institutions chargées du suivi et du contrôle des exploitations de dragage doivent disposer, à cet effet, de la logistique et des moyens nécessaires pour ce faire.

\subsection{Le sort des matériaux de dragage}

Lorsque le projet de dragage vise à la fois l'entretien des chenaux de navigation et l'exploitation su sable marin, la décision du devenir des matériaux de dragage doit se baser sur la nature et la qualité du sédiment extrait. Des seuils de pollution de sédiment doivent être établis pour décider son sort. Face au manque de textes juridiques et référentiels de qualité traitant explicitement cette matière, on peut se référer aux guides et réglementations européennes et conventions internationales. En France, l'arrêté du 14 juin 2000, accompagné d'une circulaire du Ministère chargé de l'Équipement, relative aux conditions d'utilisation du référentiel de qualité des sédiments marins ou estuariens, ainsi que des publications du GEODE et du Fond de prélèvement durable des matériaux marins (MALSF) constituent des sources d'inspiration pour définir des seuils de références déterminant le devenir des sédiments dragués (immersion, traitement et valorisation, stockage, ...). Pour la mise en dépôt à terre, on peut aussi s'inspirer des niveaux de référence prescrits par la législation française pour l'épandage des boues de stations d'épuration, définis par l'arrêté du 8 janvier 1998. Pour l'utilisation des sédiments extraits dans les travaux de construction, on peut se référer aux normes marocaines homologuées, se rapportant aux bâtiment et travaux publics. 


\section{Conclusion}

L'encadrement technique sur les plans physique, géographique, biologique et chimique des activités de dragage, que ce soit pour les besoins d'entretien ou d'exploitation est une urgence au Maroc. La bonne gouvernance environnementale de ce secteur suscite son accompagnement par des institutions de recherches et de suivis. En attendant l'approbation d'un cadre juridique et un référentiel de qualité fixant les conditions d'utilisation des sédiments, les propositions discutées dans cet article peuvent être enrichies et prises en considération dans l'évaluation des études d'impact environnementales relatives à ces activités.

\section{Références}

BOUTIMIN G. (1986). Dragage et exploitation des sables marins: qualité des matériaux et conséquences sur le milieu. Thèse, Université de Nantes, 203 p.

COOPER K., BOY S., ALDRIDGE J., REES H. (2007). Cumulative impacts of aggregate extraction on seabed macro-invertebrate communities in an area off the east coast of the United Kingdom. Journal of sea research, Vol. 57, pp 288-302. http://dx.doi.org/10.1016/j.seares.2006.11.001

CIRIA (1998). Regional seabed sediment studies and assessment of marine aggregate dredging report. Construction industry research and information association (CIRIA) publication.

DESMAZES F., MICHEL D., HOWA H., PEDREROS R. (2002). Etude morphodynamique du domaine pré-littoral nord aquitain, site atelier du TrucVert. VII ${ }^{\text {ème }}$ Journées Nationales Génie Civil- Génie Côtier, Anglet, pp 155-162. http://dx.doi.org/10.5150/jngcgc.2002.016-d

DICKSON R.R., REES J.M. (1998). Impact of dredging plumes on Race Bank and surrounding areas. CEFAS, LOWESTOFT. Unpublished Final Report to MAFF, U.K., $15 \mathrm{p}$.

DPDPM (2011). Etude d'expertise sédimentologique de la plage de Mehdia (Maroc). Rapport final, $\mathrm{n}^{\circ} 1712781,81 \mathrm{p}$.

GEODE -Groupe d'étude et d'observation sur le dragage et l'environnement- (2012). Suivis environnementaux des opérations de dragage et d'immersion. Guide méthodologique, $134 \mathrm{p}$.

HALLEMEIER R.J. (1981). A profile zonation for seasonal sand beaches from wave climate. Coastal engineering, Vol. 4, pp 253-277. http://dx.doi.org/10.1016/0378-3839(80)90022-8 HAKKOU M., BENMOHAMMADI A., CASTELLE B., BERTIN X., LABRAIMI M., EL HASSANI A., LAYACHI M. (2015). Perspectives d'optimisation technique de la gouvernance environnementale des activités du dragage du sable marin au Maroc. Bulletin de l’Institut Scientifique, Rabat, Section Sciences de la Terre, $n^{\circ} 37$. 


\section{XIV ${ }^{\text {èmes }}$ Journées Nationales Génie Côtier - Génie Civil \\ Toulon, 29 juin au $1^{\text {er }}$ juillet 2016}

HITCHCOCK D.R., BELL S. (2004). Physical impacts of marine aggregate dredging on seabed resources in coastal deposits. Journal of Coastal Research, Vol. 20(1), pp 101-114. http://dx.doi.org/10.2112/1551-5036(2004)20[101:PIOMAD]2.0.CO;2

HITCHCOCK D.R., NEWELL R.C., SEIDERER L.J. (1998). Investigation of benthic and surface plumes associated with marine aggregate mining in the United Kingdom. Report for the U.S. Department of the Interior, Bridgend Coastline Surveys Ltd, 108 p. JAUD M. (2011). Techniques d'observation et de mesure haute résolution des transferts sédimentaires dans la frange littorale Sciences de la Terre. Thèse de doctorat. Université de Bretagne occidentale, Brest, 245 p.

METL -Ministère d'équipement, du Transport et de la logistique, Maroc- (2014). Impact de la houle exceptionnelle du 6-7 janvier 2014 sur les infrastructures portuaires. NEWELL R.C., SEIDERER J.L., HITCHCOCK D.R. (1998). The impact of dredging works in coastal waters: a review of sensitivity to disturbance and subsequent recovery of biological resources on the seabed. Oceanography and marine biology: an annual review, Vol. 36, pp 127-78.

NEWELL R.C., HITCHCOCK D.R., SEIDERER L.J. (1999). Organic enrichment associated without wash from marine aggregates dredging: a probable explanation for surface sheens and enhanced benthic production in the vicinity of dredging operations. Marine pollution bulletin, Vol. 38, pp 809-818. http://dx.doi.org/10.1016/S0025-326X(99)00045-4

ROOS C.P., HULSHER S.H., DE VRIEND H.J. (2008). Modelling the morphodynamic impact of offshore sand pit geometries. Coastal engineering, Vol. 55, pp 704-715. http://dx.doi.org/10.1016/j.coastaleng.2008.02.019

TILLIN H.M., HOUGHTON A.J., SAUNDERS J.E., DRABBLE R., HULL S.C. (2011). Direct and indirect impacts of aggregate dredging. Rapport mepf/10/p144, $41 \mathrm{p}$.

VAN RIJN L., WALSTRA D.J. (2002). Morphology of pits, channels and trenches. Part I : Literature review and study approach. Report z3223, WL Delft hydraulics, $92 \mathrm{p}$.

WILLOUGHBY M.A., CRABB D.J. (1983). The behaviour of dredge generated sediment plumes in Moreton Bay. Sixth Australian conference on coastal and ocean engineering, 1983: preprints of papers. Barton, ACT: Institution of Engineers, Australie, pp 182-186. 
Thème 6 - Gestion durable des zones littorales et estuariennes 